\title{
Algoritmos para el problema de localización de plantas y centros de distribución maximizando beneficio
}

\author{
Algorithms for the problem of location of plants and \\ distribution centers maximizing Benefit \\ Luis Flores Garrido ${ }^{1 *} \quad$ Cristian Oliva San Martín ${ }^{2}$ \\ Recibido 10 de octubre de 2014, aceptado 15 de diciembre de 2015 \\ Received: October 10, 2014 Accepted: December 15, 2015
}

\begin{abstract}
RESUMEN
Este artículo presenta un caso particular del problema de localización de instalaciones. La característica de este es que considera un conjunto de clusters que representan la acumulación de clientes que demandan un producto en particular. Se desea localizar P centros de distribución donde la demanda de los clusters se satisface solo si localizamos un centro de distribución en dicho cluster. Además, se debe determinar tanto el número de plantas como sus respectivas ubicaciones con el fin de abastecer los diferentes centros de distribución localizados. El objetivo es maximizar la utilidad considerando, por un lado, los ingresos que se obtienen por satisfacer la demanda de los diferentes clusters y, por otro lado, los costos de instalación que se incurren al localizar los centros de distribución y las plantas como también los costos de transporte de material desde las plantas a los centros de distribución. Otras aplicaciones de este problema se aprecian en redes de telecomunicaciones, redes eléctricas, etc. Se presenta un nuevo modelo de programación lineal entera que permite resolver instancias pequeñas, hasta 300 clusters, en un tiempo CPU aceptable, los problemas fueron programados con AMPL, y resueltos con CPLEX 11.0. Para instancias de mayor tamaño se presentan heurísticas que permiten obtener soluciones con un GAP menor al $1 \%$.
\end{abstract}

Palabras clave: Problema de localización de instalaciones, programación lineal entera y heurística.

\begin{abstract}
This paper presents a particular case of the facility location problem. The characteristic of this problem is that it contains a set of clusters or nodes that represent the accumulation of clients who demand a single product. It is desired to locate $P$ distribution centers $(D C)$ where the demand of clusters is only satisfied if we located a distribution center in this cluster. Moreover, the number of capacitated plants and its location must be determined in order to supply the different distributions centers. The objective is to maximize the utility by considering, in one hand, the incomes that are obtained for satisfying the demand of different clusters and in the other hand, the installation costs incurred when locating the Distribution Centers and the plants as well as the costs of material transport from the plants to the distribution center. Other applications of this problem are appreciated in telecommunications networks, electrical networks, etc. The paper also present a new model of integer programming that allows to solve small instances, up to 300 clusters, in an acceptable CPU time, the problems were programmed with AMPL and solved with CPLEX 11.0. For two larger instances the authors present heuristics, which allow obtaining solutions with a GAP least than $1 \%$.
\end{abstract}

Keywords: Facility location problem, integer programming, heuristic.

1 Escuela de Postgrado. Universidad Católica de la Santísima Concepción. Alonso de Rivera 2850. Concepción, Chile. E-mail: 1flores@ing.ucsc.cl

2 Facultad de Ingeniería. Universidad Católica de la Santísima Concepción. Alonso de Rivera 2850. Concepción, Chile. E-mail: coliva@ucsc.cl

* Autor de correspondencia 


\section{INTRODUCCIÓN}

El problema de localización de instalaciones consiste en decidir la ubicación de las instalaciones para satisfacer a los clientes maximizando las utilidades. Este problema ha sido tratado por muchos autores, quienes presentan diferentes tipos de modelos pero considerando la misma función objetivo: minimizar los costos de localización y de transporte. El problema de localización no capacitado, el que no considera la capacidad de las plantas y demandas de los clientes [4] tiene restricciones del tipo $\sum y_{i j}=1$, para todo $i$ (1) y $\sum y_{i j} \leq x_{j}$, para todo $j(2)$; donde la variable $y_{i j}$ es la fracción de la demanda del cliente $i$ satisfecha por la instalación $j$ y la variable $x_{j}$ es 1 si se localiza una instalación en $j$. La restricción (1) estipula que la demanda del cliente $i$ debe ser satisfecha en su totalidad y la restricción (2) estipula que la demanda del cliente $i$ no puede ser satisfecha a partir de la instalación $j$ si no se localiza dicha instalación. En [5] se incorporan adicionalmente al problema las restricciones de capacidad y demanda: $\sum d_{i} y_{i j} \leq s_{j} x_{j}$, para todo $j$ (3) donde $s_{j}$ es la capacidad de la instalación $j$ y $d_{i}$ es la demanda del cliente $i$. La restricción (3) asegura que cada planta no puede satisfacer la demanda más allá de su capacidad. En [9] el problema de localización de plantas capacitadas se compone solamente de las restricciones (1), (2) y (3).

En el problema de localización de $p$ instalaciones capacitadas con condiciones globales [10], al objetivo de minimizar los costos de transporte entre las instalaciones y el costo de localización de estas, se agregan además los costos de producción y mano de obra. El modelo se extiende a la localización de un número $p$ de instalaciones, donde estas pueden instalarse dentro de regiones previamente determinadas al igual que los clientes. Se puede instalar un número máximo de facilidades en cada región y las plantas pueden seleccionar el tamaño de cada una de ellas. El problema de diseño de sistemas de distribución [8] consiste en un conjunto de plantas que producen múltiples productos. Estos son enviados a un conjunto de centros de distribución, los que deben satisfacer las demandas de un conjunto de clientes. El objetivo del modelo es minimizar los costos de transporte más los costos de instalación de plantas y centros de distribución con sus respectivos costos de operación. Otro problema de localización de $p$ instalaciones capacitadas se presenta en [3], el que consiste en localizar como máximo $p$ nuevas instalaciones a una línea de producción ya existente. Los criterios de localización se basan en aspectos externos como el presupuesto u otros recursos restrictivos. El objetivo del problema es minimizar los costos fijos de construcción y operación al localizar una nueva instalación en un nodo cualquiera, teniendo en cuenta que la demanda de la instalación ubicada en el nodo $j$ debe ser satisfecha completamente por la instalación inmediatamente precedente. El problema de localización de instalaciones capacitadas con múltiples productos dinámicos [7] tiene como objetivo minimizar los costos de producción, inventario, transporte, costos fijos de operación e inversión en la relocalización de facilidades considerando para esto una cadena de suministros, donde diferentes productos son entregados para satisfacer los requerimientos de múltiples puntos de demanda. El problema puede albergar diferentes tipos de instalaciones, no existiendo restricción en el número de estas ni en la cantidad de accesos usados en el tráfico. Se asume que se considerará trasladar la totalidad o parte de las instalaciones durante un horizonte de tiempo, seleccionando para esto un conjunto de sitios en los cuales nuevas instalaciones pueden ser establecidas. El problema de localización de instalaciones capacitadas en diseños de redes [5], tiene como objetivo minimizar los costos de construcción de las instalaciones y de los arcos, además de los costos de transportar unidades por los arcos, uniendo para este propósito el problema de localización capacitado y el problema de flujo de costo mínimo. Algunas extensiones al problema de localización capacitado se encuentran en [1], [2] y [11].

\section{PRESENTACIÓN DEL MODELO}

\section{Descripción del problema}

Sea una red dirigida que representa una región geográfica cualquiera, donde los nodos o cluster representan ciudades o grupos de ciudades y los arcos representan los caminos que unen estas. Supongamos que las ciudades demandan solamente un tipo de producto y si suplimos la demanda de la ciudad obtendremos ingresos proporcionales a su número de habitantes. Si localizamos un centro de distribución en un nodo $i$ suplimos la demanda de la ciudad que este representa pero, a su vez, debemos abastecerla de productos, para esto se deben instalar 
las plantas capacitadas suficientes con tal de suplir los requerimientos del centro de distribución. No se puede localizar una planta en una ciudad que ya tiene albergado un centro de distribución. Una planta puede abastecer a más de un centro de distribución a la vez y estos pueden ser abastecidos por más de una planta. Los costos asociados a la localización pueden ser de construcción y/o mantención, estos difieren según cada ciudad y pueden ser distintos para las plantas y los centros de distribución. El costo de transporte corresponde al envío de productos desde las plantas hasta los centros de distribución.

- El modelo tiene en cuenta lo siguiente:

- Cada nodo representa un punto de demanda.

- Este punto de demanda se satisface solo si se instala un centro de distribución.

- Las plantas y centros de distribución solo se pueden localizar en los nodos.

- Una vez localizado un centro de distribución su demanda debe ser suplida por una o más plantas.

- Una planta puede suplir la demanda de más de un centro de distribución a la vez.

- Se puede instalar solamente una planta o un centro de distribución por nodo.

- Los arcos pueden ser o no capacitados.

\section{Formulación del problema}

Sea $G=(N, A)$ una red definida por un conjunto $N$ compuesto por $n$ nodos y un conjunto $A$ compuesto por $\mathrm{m}$ arcos unidireccionales. Cada nodo $j$ tiene asociado un costo de localización de plantas $f_{j}$, un costo de localización de centros de distribución $c_{j}$, una capacidad $q_{j}$, un ingreso por unidad de producto $g_{j}$, una demanda $d_{j}$. Cada arco $(i, j)$ tiene asociado un costo de envío $h_{i j}$. La formulación para el problema es la siguiente:

\section{Conjuntos}

$N=\{1,2, \ldots, n\}$ el conjunto de nodos que pertenecen a la red.

$A$ el conjunto de $\operatorname{arcos}(i, j)$ que pertenecen a la red.

\section{Parámetros}

$c_{j}$ el costo de localizar y/o mantener un centro de distribución en el nodo $j$.

$f_{j}$ el costo de localizar y/o mantener una planta en el nodo $j$.

$d_{j}$ la demanda del centro de distribución en el nodo $j$. $q_{j}$ la capacidad de una planta en el nodo $j$. $g_{j}$ ingreso por unidad de producto en el nodo $j$. $h_{i j}$ el costo por enviar una unidad de producto por el $\operatorname{arco}(i, j)$.

$p$ la cantidad de centros de distribución que se desea localizar.

$a_{i j}=\{1$, si existe arco desde el nodo $i$ al nodo $j .0$, en otro caso $\}$.

\section{Variables}

$y_{j}$ la cantidad de producto que sale desde un nodo $j$. $x_{i j}$ unidades del producto enviadas desde el nodo $i$ hasta el nodo $j$.

$z_{j}=\{1$, si se localiza un centro de distribución en el nodo $j$. 0 , en otro caso $\}$.

$w_{j}=\{1$, si se localiza una planta en el nodo $j .0$, en otro caso $\}$

Definidas las variables, la formulación es la siguiente:

$\max \sum_{j \in N}\left(g_{j} d_{j}-c_{j}\right) z_{j}-\left(\sum_{j \in N} f_{j} w_{j}+\sum_{i \in N} \sum_{j \in N} h_{i j} x_{i j}\right)$

$\mathrm{s} / \mathrm{a}$

$\sum_{j \in N} z_{j}=p$

$\mathrm{W}_{\mathrm{j}}{ }^{+} \mathrm{z}_{\mathrm{j}} \leq 1 \quad \forall \mathrm{j} \in \mathrm{N}$

$y_{j} \leq q_{j} w_{j} \quad \forall j \in N$

$\sum_{i \in N} a_{i j} x_{i j}-\sum_{i \in N} a_{j i} x_{j i}=d_{j} z_{j}-y_{j} \quad \forall j \in N$

$z_{j} \in\{0,1\}$

$w_{j} \in\{0,1\}$

$x_{i j} \in Z_{0}^{+}$

$y_{j} \in Z_{0}^{+}$

La ecuación (1) maximiza la ganancia obtenida por la venta de producto, esto lo consigue maximizando los ingresos y minimizando los costos de localización y/o mantención de los centros de distribución y plantas (localiza un mínimo de plantas para suplir la demanda), además de los costos de transporte entre estas. La restricción (2) nos indica la cantidad de centros de distribución que deseamos localizar. El conjunto de restricciones (3) nos indica que solo 
podemos localizar a lo más una planta o un centro de distribución por nodo. El conjunto de restricciones (4) nos indica que si se instala una planta en el nodo $j$, de esta podrá salir una cantidad máxima de $q j$ unidades de productos. El conjunto de restricciones (5) nos entrega la igualdad de flujo. Por ejemplo, si se instala un centro de distribución en el nodo $j$ $\left(z_{j}=1\right)$ entonces debe satisfacerse la demanda de ese centro de distribución a partir de alguna planta. Si se instala una planta en el nodo $j\left(w_{j}=1\right)$ entonces se enviará desde $j$ una cantidad $y_{j}$ para satisfacer la demanda de centros de distribución.

A continuación ilustramos un pequeño ejemplo de una red de 10 nodos y 14 arcos con los parámetros mostrados en la Tabla 1. Se requiere, como dato del problema, instalar $p=2$ centros de distribución.

Tabla 1. Parámetros

\begin{tabular}{|c|r|r|r|r|r|r|r|r|r|r|}
\hline $\boldsymbol{i}$ & $\mathbf{1}$ & $\mathbf{2}$ & $\mathbf{3}$ & $\mathbf{4}$ & $\mathbf{5}$ & $\mathbf{6}$ & $\mathbf{7}$ & $\mathbf{8}$ & $\mathbf{9}$ & \multicolumn{1}{c|}{$\mathbf{0}$} \\
\hline$q:$ & 74 & 108 & 129 & 60 & 84 & 78 & 54 & 77 & 143 & 96 \\
\hline $\mathrm{d}:$ & 83 & 63 & 41 & 10 & 63 & 75 & 88 & 34 & 33 & 87 \\
\hline$c:$ & 1.416 & 1.822 & 1.329 & 1.679 & 1.790 & 1.074 & 1.914 & 1.492 & 1.349 & 1.469 \\
\hline$f:$ & 1.215 & 1.644 & 1.711 & 1.980 & 1.351 & 1.268 & 1.851 & 1.588 & 1.570 & 1.580 \\
\hline $\mathrm{g}:$ & 93 & 91 & 40 & 32 & 55 & 83 & 98 & 87 & 50 & 40 \\
\hline
\end{tabular}

$$
h_{i j}=\left(\begin{array}{cccccccc}
1 & & 2 & & & & & 1 \\
& 2 & & & & & & \\
& & 2 & & 2 & & & \\
& & & 1 & & & 2 & \\
& & & & 1 & 2 & & 2 \\
& & & & & 2 & & 2 \\
& & & & & & 2 &
\end{array}\right)
$$

La fila 1 de la Tabla 1 muestra el identificador de cada nodo. La fila 2 de la misma tabla, presenta la capacidad que la planta tendría en el caso de instalarse en el nodo respectivo. Las filas denotadas por $d, c$, $f$ y $g$ presentan la demanda, costo de localizar un centro de distribución, costo de instalar una planta y los ingresos por unidad obtenidos en cada nodo, respectivamente.

La Figura 1 muestra la solución óptima del problema. El número de plantas localizadas es 2 con un beneficio total de \$ 9.994. Los centros de distribución fueron localizados en nodos 1 y 7 ; y las plantas en los nodos 6 y 9 . Observe que la planta localizada en el nodo 6 produce 78 unidades físicas lo que equivale a su capacidad máxima de producción. Sin embargo, la planta localizada en el nodo 9, que tiene capacidad máxima de 143, solo produce 93 unidades físicas por unidad de tiempo. Esta planta atiende ambos centros de distribución.

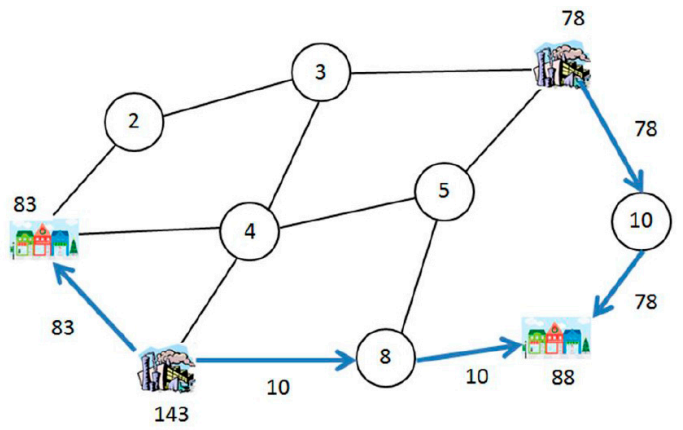

Figura 1. Solución óptima del ejemplo.

\section{EXPERIENCIA COMPUTACIONAL}

Con el fin de estudiar los tiempos computacionales (tiempo CPU) del modelo de localización de plantas y centros de distribución maximizando beneficio se crearon instancias similares a los utilizados en [6]. Se crearon redes de 100, 200 y 300 nodos cada una con $9.900,39.800$ y 89.700 arcos dirigidos, respectivamente. Las redes se crearon utilizando números aleatorios, para la capacidad de las plantas se utilizó una distribución uniforme $(50,200)$, para la demanda una distribución uniforme $(0,100)$, para los costos de mantención de las plantas y los centros de distribución se utilizó una distribución uniforme $(1.000,2.000)$, los ingresos por venta de unidad de producto se generaron mediante una distribución uniforme $(200,250)$, y el ingreso total en el nodo $i$ se calculó como $I_{i}=g_{i} * d_{i}$.

Los costos por enviar una unidad de producto por el $\operatorname{arco}(i, j)$ se define como $h_{i j}=u^{*} t_{i j}$, donde $t_{i j}$ se distribuye uniforme $(1,10)$, con $u=2$ para redes con costos de transporte bajos y $u=10$ para redes con costos de transporte altos.

Cada instancia creada está en función del número de nodos $(n)$, del tipo de costos de transporte $(u)$ y del número de centros de distribución a localizar $(p)$. Por cada combinación de estos parámetros se crearon 100 instancias distintas. Hay 18 combinaciones posibles. En particular, para $n=100$ y $u=2$ se crearon 300 instancias ( 100 por cada $\mathrm{P}$ ) pero se debe tener en cuenta que tanto para $p=5, p=6$ y $p=7$ los ingresos, costos, capacidades y demandas son idénticos. $\mathrm{La}$ Tabla 2 resume lo expresado anteriormente. 
Tabla 2. Experimentos.

\begin{tabular}{|c|c|c|}
\hline Factor & Número de niveles & Valor niveles \\
\hline$n$ & 3 & $100,200,300$ \\
\hline$|A|$ & 1 & $\mathrm{n} *(\mathrm{n}-1)$ \\
\hline$p$ & 3 & $5,6,7$ \\
\hline$u$ & 2 & 2,10 \\
\hline
\end{tabular}

\section{Resultado}

El modelo se programó utilizando AMPL y los problemas se resolvieron de manera óptima utilizando las librerías de resolución de ILOG CPLEX 11.0, para esto se utilizó un computador con un procesador Intel ${ }^{\circledR}$ CoreTM 2 Duo de $2.66 \mathrm{GHz}, 2 \mathrm{~GB}$ de memoria RAM y con sistema operativo Windows $\mathrm{XP}$ profesional.

La Tabla 3 muestra el tiempo promedio (desviación estándar) utilizado para encontrar la solución óptima de las 18 combinaciones, cada una de 100 problemas test. Para las redes cuyos costos de transporte son altos $(u=10)$ el tiempo promedio de CPU aumenta para un mismo $p$ al aumentar el número de nodos en la red.

Tabla 3. Tiempos (segundos) medios de CPU.

\begin{tabular}{|c|r|c|c|c|}
\hline \multicolumn{2}{|c|}{ Nodos } & \multicolumn{3}{|c|}{ Instalaciones } \\
\hline$|\boldsymbol{N}|$ & $\boldsymbol{u}$ & $\boldsymbol{p}=\mathbf{5}$ & $\boldsymbol{p}=\mathbf{6}$ & $\boldsymbol{p}=\mathbf{7}$ \\
\hline 100 & 2 & $1,38(1,10)$ & $3,93(5,05)$ & $2,09(2,24)$ \\
& 10 & $0,86(0,55)$ & $1,11(0,60)$ & $1,34(1,14)$ \\
\hline 200 & 2 & $19,11(18,84)$ & $81,30(124,73)$ & $27,09(32,01)$ \\
& 10 & $8,79(7,44)$ & $11,59(8,89)$ & $15,07(11,29)$ \\
\hline 300 & 2 & $89,96(83,96)$ & $516,06(705,80)$ & $129,22(127,59)$ \\
& 10 & $39,19(32,05)$ & $59,07(43,44)$ & $80,43(64,49)$ \\
\hline
\end{tabular}

Si aumentamos $p$ manteniendo el número de nodos de la red, el tiempo de CPU también aumenta, notándose una mayor desviación estándar en aquellas redes que constan de 300 nodos. Para las redes cuyos costos de transporte son bajos $(u=2)$, si mantenemos el número $p$ fijo el tiempo promedio de CPU aumenta si el número de nodos de la red también aumenta. Si mantenemos el número de nodos de la red fijo y aumentamos el número de $p$ podemos observar que para $p=6$ el tiempo promedio de CPU es mayor que para $p=5$ y $p=7$, esto independiente del número de nodos de la red. Es necesario indicar que no existe una relación directa entre el número de centros de distribución a instalar y el número de plantas, pues estas dependen de la cantidad demandada y de la oferta de cada nodo.
Para las redes cuyos costos de transporte son bajos $(u=2)$ el tiempo promedio de CPU es mayor que para aquellas redes cuyos costos de transporte son altos $(u=10)$ independientes del número de centros de distribución que se deban localizar $(p)$, esto se cumple para redes compuestas por 100, 200 y 300 nodos. Como se observa en la Tabla 3, los tiempos de CPU requerido para resolver el problema de localización de plantas y centros de distribución maximizando beneficio son relativamente pequeños, el máximo tiempo promedio de CPU observado es de 516,60 segundos; sin embargo, para problemas de mayor complejidad el esfuerzo computacional es alto. Para observar el tiempo de CPU en problemas de mayor complejidad se creó una red del 1.000 nodos y 999.000 arcos dirigidos, el tiempo de CPU requerido para resolver este problema de forma óptima fue de 26.164,8 segundos, se espera que para redes aún mayores el esfuerzo computacional requerido para resolver el problema de localización de plantas y centros de distribución maximizando beneficio sean mayores.

\section{HEURÍSTICAS}

\section{Presentación Heurísticas}

Como se observa en la Tabla 3, el tiempo promedio de CPU aumenta mientras mayor sea el número de nodos, debido a esto se presentan dos heurísticas con el fin de disminuir el esfuerzo computacional; sin embargo, la aplicación de estas no asegura encontrar la solución óptima. Una de ellas es de tipo golosa (HG) y otra reduce el problema aplicando un radio de cobertura para eliminar variables (HEV).

En primer lugar, la HG determina la localización de los $p$ centros de distribución, la elección se basa en escoger los $p$ centros de distribución cuyos ingresos netos (ganancia-costo de instalación) sean mayores. En segundo lugar, se debe determinar la localización de las plantas, el criterio de elección se basa en escoger aquellos nodos cuya razón, entre el costo y la capacidad, sean menores excluyendo los nodos elegidos como centros de distribución; la elección se realizará hasta que la suma de las ofertas de las plantas sea mayor a la suma de la demanda de los centros de distribución. En tercer lugar, se debe determinar el costo de envío de productos desde las plantas hasta los centros de distribución, para esto resolveremos el problema de localización 
de plantas y centros de distribución maximizando beneficio, eliminando las restricciones (6) y (7), y utilizando como solución para las localizaciones de las plantas y los centros de distribución los nodos encontrados anteriormente. El procedimiento de HG se describe a continuación.

Paso 0:

Inicializar $d t=0$ y $o t=0$

Paso 1:

Para $(k=1$ hasta $k=p)$

Hacer $J=\operatorname{argmax}_{\mathrm{i} \in \mathrm{N}}(g(i)-c(i))$

Hacer $d t=d t+d(J)$

Paso 2:

Mientras $(d t>o t)$

Hacer $I=\operatorname{argmin}_{\mathrm{i} \in(\mathrm{N}-\mathrm{J})}(f(i) / q(i))$

Hacer $o t=o t+q(I)$

Paso 3:

Resolver el problema

$$
f o=\max \sum_{i \in J}(g(i)-c(i))-\sum_{i \in N} \sum_{j \in N} h_{i j} x_{i j}-\sum_{i \in I} f(i)
$$

s/a

$y_{j}=0 \quad \forall j \in(N-I)$

$y_{j} \leq q_{j} w_{j} \quad \forall j \in N$

$\sum_{i \in N} a_{i j} x_{i j}-\sum_{i \in N} a_{j i} x_{j i}=d_{k} z_{k}-y_{l} \quad \forall j \in N, k \in J, l \in I$

Solución heurística fo

La HEV determina la localización de los $p$ centros de distribución y de las plantas de la misma manera que la HG. Luego obtenemos la distancia o costo mínimo desde cada planta a cada centro de distribución. Con la mayor de estas mínimas distancias se genera un indicador de eliminación de arcos. Como las localizaciones escogidas para las plantas son las mejores en relación costo/ capacidad y las localizaciones escogidas para los centros de distribución son las mejores en relación a la ganancia entregada, se evita explorar nodos que no son atractivos para la solución, es el caso de nodos muy alejados de aquellos centros de distribución que entregan altos beneficios, esto se realiza eliminando los arcos cuyas distancias o costos son mayores a la distancia máxima escogida con anterioridad. El paso siguiente es resolver el problema de localización de plantas y centros de distribución maximizando beneficio utilizando como solución para la localización de los centros de distribución los nodos encontrados anteriormente y aquellos arcos que no han sido eliminados. Por último computamos la solución. El procedimiento de la HEV se describe en este artículo como:

Paso 0:

Inicializar $d t=0$, ot $=0, t=0$

Paso 1:

Para $(k=1$ hasta $k=p)$

Hacer $J=\operatorname{argmax}_{\mathrm{i} \in \mathrm{N}}(g(i)-c(i))$

Hacer $d t=d t+d(J)$

Paso 2:

Mientras $(d t>o t)$

Hacer $I=\operatorname{argmin}_{\mathrm{i} \in(\mathrm{N}-\mathrm{J})}(f(i) / q(i))$

Hacer $o t=o t+q(I)$

Paso 3

Para $(w=1$ hasta $w=k)$

Para $(c=1$ hasta $c=p)$

Resolver

$f o=\min \sum_{i \in N} \sum_{j \in N} h_{i j} x_{i j}$

s/a

$\sum_{i \in N} x_{i j}-\sum_{j \in N} x_{j i}=\left\{\begin{array}{cr}1 & \forall j \in I \\ 0 & \forall j \notin(I \cup J) \\ -1 & \forall j \in J\end{array}\right.$

$\mathrm{Si}(\mathrm{t} \leq \mathrm{fo})$

Hacer $t=$ fo

Paso 4:

$\mathrm{Si}(h i j<\mathrm{t} \wedge \wedge h i j \neq 0)$

Hacer $a_{i j}=1$

Si no

Hacer $a_{i j}=0$

Paso 5:

Resolver el problema

$f o=\max \sum_{i \in J}(g(i)-c(i))-\sum_{i \in N} \sum_{j \in N} h_{i j} x_{i j}-\sum_{i \in I} f(i)$ 
s/a

$$
\begin{aligned}
& y_{j}=0 \quad \forall j \in(N-I) \\
& y_{j} \leq q_{j} w_{j} \quad \forall j \in J \\
& \sum_{i \in N} a_{i j} x_{i j}-\sum_{i \in N} a_{j i} x_{j i}=d_{k} z_{k}-y_{j} \quad \forall j \in N, k \in J \\
& w_{j}{ }^{+} z_{j} \leq 1 \quad \forall j \in(N-J)
\end{aligned}
$$

\section{Solución heurística fo}

\section{Análisis heurísticas}

El análisis de las heurísticas se realizó utilizando las redes creadas para desarrollar la sección EXPERIENCIA COMPUTACIONAL. Las heurísticas se programaron con AMPL y resueltas utilizando las librerías de ILOG, para esto se utilizó un computador con un procesador Intel® CoreTM 2 Duo de 2.66 Ghz, 2 GB de memoria RAM y con sistema operativo Windows XP profesional. Para cuantificar el error se utilizó un GAP, este mide en forma porcentual el error existente entre la solución óptima y la solución encontrada por la heurística, $\mathrm{GAP}=\left(\left|\mathrm{z}^{*}-\mathrm{HS}\right| / \mathrm{HS}\right)^{*} 100 \%$.

La Tabla 4 muestra el tiempo promedio utilizado para encontrar la solución óptima de las 18 combinaciones de forma óptima y utilizando las heurísticas, el GAP de las heurísticas.

Para saber el comportamiento de las heurísticas en cuanto al tiempo de CPU, y el comportamiento del error existente entre la solución óptima y la encontrada por las heurísticas, se realizaron dos análisis. El primer análisis se realizó variando el número de nodos de la red manteniendo fijo el número de centros de distribución localizados. Para el segundo análisis se mantuvo fijo el número de nodos variando el número de centros de distribución. El tiempo de CPU, de ambas heurísticas, aumenta progresivamente al aumentar el número de nodos del problema, esto debido a que la complejidad del problema aumenta con el número de nodos. Además los tiempos promedios de CPU requeridos por la HEV son mayores que los tiempos promedios requeridos por la $\mathrm{HG}$.

El tiempo medio de CPU utilizado por la HEV para encontrar las soluciones, manteniendo fijo el número

\begin{tabular}{|c|c|c|c|c|c|c|c|}
\hline \multicolumn{3}{|c|}{ Característica } & \multirow{2}{*}{ 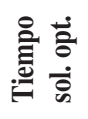 } & \multirow{2}{*}{ ڤ્己 } & \multirow{2}{*}{ 总泀 } & \multirow{2}{*}{ 氙 } & \multirow{2}{*}{ ¿ } \\
\hline Nodos & $u$ & $p$ & & & & & \\
\hline \multirow[t]{3}{*}{100} & 2 & 5 & 1,38 & 0,130 & 1,43 & 0,46 & 0,072 \\
\hline & & 6 & 3,93 & 0,132 & 2,70 & 0,57 & 0,096 \\
\hline & & 7 & 2,09 & 0,135 & 2,51 & 0,39 & 0,078 \\
\hline \multirow[t]{3}{*}{100} & 10 & 5 & 0,86 & 0,132 & 1,22 & 3,62 & 0,160 \\
\hline & & 6 & 1,11 & 0,129 & 1,73 & 3,37 & 0,184 \\
\hline & & 7 & 1,34 & 0,134 & 2,18 & 3,02 & 0,199 \\
\hline \multirow[t]{3}{*}{200} & 2 & 5 & 19,11 & 0,484 & 6,16 & 0,49 & 0,048 \\
\hline & & 6 & 81,30 & 0,490 & 18,92 & 1,56 & 0,066 \\
\hline & & 7 & 27,09 & 0,491 & 10,40 & 0,40 & 0,053 \\
\hline \multirow[t]{3}{*}{200} & 10 & 5 & 8,79 & 0,486 & 4,09 & 3,42 & 0,228 \\
\hline & & 6 & 11,59 & 0,487 & 5,70 & 3,18 & 0,168 \\
\hline & & 7 & 15,07 & 0,503 & 7,20 & 2,83 & 0,119 \\
\hline \multirow[t]{3}{*}{300} & 2 & 5 & 89,96 & 1,298 & 19,02 & 0,54 & 0,108 \\
\hline & & 6 & 516,06 & 1,287 & 77,12 & 0,63 & 0,120 \\
\hline & & 7 & 129,22 & 1,318 & 31,98 & 0,43 & 0,105 \\
\hline \multirow[t]{3}{*}{300} & 10 & 5 & 39,19 & 1,308 & 10,61 & 3,25 & 0,216 \\
\hline & & 6 & 59,07 & 1,289 & 15,35 & 3,09 & 0,197 \\
\hline & & 7 & 80,43 & 1,320 & 21,51 & 2,86 & 0,199 \\
\hline
\end{tabular}
de nodos del problema, aumenta con el número de
Tabla 4. Tiempo (segundos) medios de CPU, GAP promedio (\%).

instalaciones. Al igual que los tiempos promedios de CPU utilizados para encontrar la solución óptima, el tiempo promedio de CPU es mayor en aquellas redes cuyos costos de arcos son bajos $(u=2)$ observando además un aumento notorio para aquellos problemas cuyo número de instalaciones es 6 . El comportamiento del GAP generado al utilizar la heurística Golosa cuando se varía el número de instalaciones y se mantiene fijo el número de nodos para los problemas cuyos costos de arcos son altos $(u=10)$ aumenta, es decir, la calidad de la solución entregada por la heurística golosa va a estar sujeta a los costos de los arcos, si éstos costos aumentan mayor será el GAP generado, esto se debe a que el criterio de selección de las localizaciones de plantas se realiza sin tener en cuenta los costos de los arcos, además el ahorro que significa localizar plantas en aquellos nodos que entregan la mejor relación costo/capacidad se pierde, debido a que muchos de los arcos que unen estos nodos tienen costos altos. Por otro lado, la HEV solamente utiliza como solución la localización de los centros de distribución dejando que el modelo genere la localización de las plantas encontrando así mejores soluciones, debido a esto el GAP generado al utilizar la heurística con eliminación de variables no está sujeto al costo de los arcos, entregando mejores soluciones. 
La calidad de la solución encontrada por la HEV es mejor en comparación con la HG, esto se hace notorio para redes cuyos costos de transporte son altos $(u=10)$, además se observa que la HEV entrega soluciones cuyos GAP generados no superan el $1 \%$. Cabe señalar que al igual que la HG, los GAP de la HEV aumentan si el problema tiene altos costos de transporte $(u=10)$.

Además, podemos mencionar que para el caso de 1.000 nodos el tiempo de ejecución de la heurística fue de 1,437 segundos con un valor de la mejor solución encontrada de 104.090. La solución óptima de la instancia es 108.863 lo que indica que el GAP del valor asociado a la solución heurística respecto a la solución óptima es de $4.413 \%$.

\section{CONCLUSIONES}

Este trabajo presenta un modelo de programación entera mixta que genera una extensión del clásico modelo de localización de facilidades capacitadas para la localización de dos tipos de entidades dentro de una red. Este problema tiene un gran número de aplicaciones en redes de suministros, telecomunicaciones y otras.

En este trabajo se propusieron dos heurísticas para resolver los problemas. El primer método consiste en asignar la localización de las plantas y los centros de distribución; el segundo, consiste en asignar la localización de los centros de distribución y generar un radio de cobertura para eliminar variables del problema, estos dos métodos permiten resolver problemas con un nivel de calidad deseado. Los tiempos computacionales utilizados por la Heurística Golosa son extremadamente pequeños; sin embargo, para redes cuyos costos de arcos son altos las soluciones entregadas por este pierden calidad. La heurística de eliminación de variables encuentra buenas soluciones, sin importar el costo de los arcos, pero con tiempos computaciones considerablemente mayores a los utilizados por la Heurística Golosa, pero mucho menores a los utilizados obtener la solución óptima. Comparando los métodos podemos asegurar que la Heurística de Eliminación de Variables es mejor que la Heurística Golosa en cuanto al nivel de calidad de la solución; sin embargo, la Heurística Golosa es mejor en cuanto al tiempo computacional.
Finalmente, se recomienda el uso de las heurísticas para tamaños superiores a los 1.000 nodos pues sus tiempos computaciones son del orden de unos cuantos segundos en comparación con un algoritmo exacto que toma aproximadamente siete horas de ejecución.

\section{AGRADECIMIENTOS}

Artículo parcialmente financiado por DIN 112008 de la Universidad Católica de la Santísima Concepción.

\section{REFERENCIAS}

[1] K. Aardal, Y. Pochet and L. Wosley. "Capacitated Facility Location: Valid Inequalities and Facets". Mathematics of Operations Research. Vol. 20, Issue 3, pp. 562-582. 1995. ISSN: 0364-765X.

[2] K. Aardal. "Reformulation of Capacitated Facility Location Problems: How Redundant Information can Help". Annals of Operations Research. Vol. 82, pp. 289-308. 1998. ISSN: 1572-9338.

[3] J. Brimberg, E. Korach, M. Eben-Chaim and A. Mehrez. "The Capacitated p-Facility Location Problem on the Real Line". International Transaction in Operational Research. Vol. 8, Issue 6, pp. 727-738. 2001. ISSN: 0969-6016.

[4] M. Daskin. "Network and Discrete Location: models, algorithms and applications". Vol. 1. 1995. Wiley Interscience Publication. ISBN: 9781118032343.

[5] A. Klose and A. Drexl. "Facility Location Models for Distribution System Design". European Journal of Operational Research. Vol. 162, Issue 1, pp. 4-49. 2005. ISSN: 0377-2217.

[6] S. Melkote and M. Daskin. "Capacitated Facility Location/Network Design Problems". European Journal of Operational Research. Vol.129, Issue 3, pp. 481- 495. 2001. ISSN: 0377-2217.

[7] M.T. Melo, S. Mickel and F. Saldanha da Gama. "Dynamic Multi-Commodity Capacitated Facility Location: A Mathematical Modeling Framework for Strategic Supply Chain Planning". Computers \& Operations Research. Vol. 33, Issue 1, pp. 181-208. 2006. ISSN: 0305-0548. 
[8] H. Pirkul and V. Jayaraman. "A Multi Commodity, Multi - Plant, Capacitated Facility Location Problem: Formulation and Efficient Heuristic Solution”. Computers \& Operations Research. Vol. 25, Issue 10, pp. 869-878. 1998. ISSN: 0305-0548.

[9] R. Sridharan. "The Capacitated Plant Location Problem". European Journal of Operational Research. Vol. 87, Issue 2, pp. 203-213. 1995. ISSN: 0377-2217.
[10] S.S. Syam. "A Model for the Capacitated p-Facility Location Problem in Global Environments". Computers \& Operations Research. Vol. 24, Issue 11, pp. 1005-1016. 1997. ISSN: 0305-0548.

[11] L. Wu, X. Zhang and J. Zhang. "Capacitated Facility Location Problem With General Setup Cost". Computer \& Operations Research. Vol. 33, Issue 5, pp. 1226-1241. 2006. ISSN: 0305-0548. 